\title{
Attentional spread in the statistical processing of visual displays
}

\author{
SANG CHUL CHONG and ANNE TREISMAN \\ Princeton University, Princeton, New Jersey
}

\begin{abstract}
We tested the hypothesis that distributing attention over an array of similar items makes its statistical properties automatically available. We found that extracting the mean size of sets of circles was easier to combine with tasks requiring distributed or global attention than with tasks requiring focused attention. One explanation may be that extracting the statistical descriptors requires parallel access to all the information in the array. Consistent with this claim, we found an advantage for simultaneous over successive presentation when the total time available was matched. However, the advantage was small; parallel access facilitates statistical processing without being essential. Evidence that statistical processing is automatic when attention is distributed over a display came from the finding that there was no decrement in accuracy relative to single-task performance when mean judgments were made concurrently with another task that required distributed or global attention.
\end{abstract}

As we navigate through the environment, we sometimes focus attention on a single object, such as a tree, and sometimes spread it over a wider area to see the forest. The forest, in turn, can yield two different kinds of information. It may have global properties of its own, such as a shape, a color, an orientation, and a density, and it also comprises the aggregate properties of its individual elements, which can be statistically summarized. The individual trees have a mean size with a particular variance and range, a mean separation, a mean leafiness, and so on. Most environments are hierarchically structured, as in this example. Perceptual mechanisms have evolved to form representations that connect objects hierarchically, giving properties of the wholes, as well as properties of their component parts. We perceive the global features that emerge from the arrangement of elements, the individual properties of particular local elements, and the statistical properties of the ensemble. How do we extract these three kinds of information - global, local, and statistical?

A possible hypothesis, some aspects of which we explore in this article, is that there are different modes of processing and that each is favored by a particular distribution of attention. At one extreme, when attention is focused on a single object, we bind its features, determine its structure, and perhaps identify it. At the other extreme, we may attend globally to the scene as a whole. With attention set to the global scale, we can access the gist, or semantic interpretation, of the scene (for example, a beach in Summer, a modern kitchen) and its global layout. When attention is distributed over a set of items

This research was supported by NIH Grant 1 RO1 MH58383, by Israeli Binational Science Foundation Grant 1000274, and by Conte Center Grant MH062196. Correspondence concerning this article should be addressed to S. C. Chong, Department of Psychology, Vanderbilt University, 301 Wilson Hall, 111 21st Avenue South, Nashville, TN 37203 (e-mail: sangchul.chong@vanderbilt.edu). but the scale is adjusted to that of individual elements, we have access to their statistical properties. Between these extremes, we may attend to pairs or triplets of objects to determine the relations between them-for example, the book is under the table, Mary is stroking the cat. The apparently complete and veridical representation of the surrounding scene that we normally experience may be an illusion generated from occasional detailed samples, together with statistical summaries of remaining areas and an overall interpretation of the meaning or gist. This sampling and summarizing at different levels of representation may help account for the striking change blindness recently explored by Rensink (2002), Simons (Simons \& Levin, 1997), and others.

\section{Global Versus Local Properties}

In research pioneered by Navon (1977), global and local processing of shape have been compared, using large shapes made of smaller shapes. These paradigms varied both the size of the attended area and the scale of the attended object. An early issue concerned the order of processing: Is the overall structure identified earlier in time than the component parts? Navon reported evidence supporting global precedence. Global precedence, in a different sense, is also reflected in the asymmetry of interference between global and local forms: A global form typically creates greater interference in the processing of a local form than the converse (Hoffman, 1980; Miller, 1981; Pomerantz, 1983).

A second issue was whether there are separate systems for global and local processing and how these systems are distributed in the two cerebral hemispheres. Hemispheric specialization suggesting global processing in the right brain and local processing in the left was found in unilateral brain-damaged patients (Delis, Robertson, \& Efron, 1986), and in normal participants with fMRI 
(Fink et al., 1996) and with ERPs (Heinze, Hinrichs, Scholz, Burchert, \& Mangun, 1998). Significant increases in relative $\mathrm{rCBF}$ were seen in the right lingual gyrus when participants attended to global forms and in the left inferior occipital cortex when they attended to local forms. The N2 component of ERPs (260-360 msec) elicited by a hierarchical letter showed a larger response with attention to the local letters in the left hemisphere and to the global letter in the right, but earlier components showed no lateral asymmetries.

A final question considered in this line of research has been whether it is possible to access both global and local information at the same time. Here, the results are more complex and may depend on the nature of the task. Using alphanumeric stimuli, Farell and Pelli (1993) found no effect on the accuracy of identifying a target letter or digit when they mixed scales within the same display. On the other hand, Ward (1982) found a delay when participants had to switch the level to which they were attending within a hierarchical stimulus. Similarly, Robertson, Egly, Lamb, and Kerth (1993) showed that a target letter was found more quickly when the level at which it would appear was cued in advance. Using a stimulus containing many different local directions of motion, Watamaniuk and McKee (1998) found that participants could either form a unified global percept of motion in the direction of the mean or focus on one local direction of motion. Direction discrimination thresholds in the postcued condition were not significantly higher than those obtained in the precued condition, suggesting that direction information for both global and local motion is encoded in parallel.

\section{Statistical Versus Individual Processing of Local Elements}

Studies with hierarchical stimuli confound two senses of the term global attention: the spatial spread of attention over a wide, rather than a narrow, area and the selection of elements defined at the larger, rather than the smaller, spatial scale (or the whole, rather than a part). These two aspects usually go together in tests with hierarchical stimuli, but they need not do so. The environment is full of sets, such as the trees in a forest, the grass in a field, a flock of birds, the cars in a parking lot, about which we are unlikely to store individuating information. We can perceive them either as wholes with emergent global properties of shape and layout or as sets of local elements, with a mean value and a variance on a number of different dimensions and with other statistical properties, such as homogeneity or heterogeneity. Our interest in this article is to explore the statistical processing of sets of elements, rather than the global processing of the ensemble, and to relate this processing to the optimal deployment of attention. To distinguish processing of multiple elements as a set from processing of global properties of the whole, we will use the term distributed attention (as opposed to global attention). This is the perceptual mode in which we suggest that statistical properties are coded. We test this hypothesis by keeping the scale of the relevant elements constant, varying whether attention en- compasses one element or many, and then measuring thresholds for judging the mean size of the set.

Statistical processing of visual displays has been studied in the context of natural images (Field, 1987; Geisler, Perry, Super, \& Gallogly, 2001) and of texture perception (Haralick, Shanmugam, \& Dinstein, 1973; Julesz, 1981), but not much research has involved directly comparing the coding of individual elements with the coding of sets. Treisman and Gormican (1988; Treisman, 1991) used the idea of statistical coding in the context of visual search, suggesting that pop-out performance depends on distributed attention to the display as a whole. The idea was that preattentive processes pool feature information within each map in a set of coarsely coded feature maps, giving an average measure of the degree to which each of these feature values is present in the display. A unique target is detected if it generates activity in a set of detectors that are not also activated by the distractors. When the target has no unique feature and activates the same detectors as the distractors, but to a lesser degree, an attention window of adjustable size is narrowed sufficiently to isolate pooled samples whose averaged signal differs detectably when the target is present in the sample and when it is not. Thus, a continuum from serial to parallel search can be generated by varying the size of the attention window to match the statistical properties of the display.

Previous experiments also illustrate some effects of different attentional modes. When attention is broadly spread over the display, globally coherent motion is perceived (group motion for the Ternus stimulus), whereas when attention is focused on a small area of the display, local motion is perceived (oscillatory motion, or element motion for the Ternus stimulus; Hock, Park, \& Schöner, 2002). Watamaniuk and Sekuler (1992) found that global motion discrimination improved as the area of the display increased, suggesting that simultaneously accessing more information helps us to process the statistical properties of the moving elements. Austin and Enns (2000) found that global change was more easily detected than local change when attention was distributed among several hierarchical stimuli.

Ariely (2001; Ariely \& Burbeck, 1995) was one of the first explicitly to explore the ability to extract the statistics of a display. He showed that mean size is perceived more accurately than individual sizes in a display of disks of varied sizes and that there is little effect of the number of disks. Chong and Treisman (2003) used Ariely's paradigm to show that judgments of mean size are almost as accurate as judgments of single items presented alone and that they are little affected either by exposure duration or by delay, suggesting an automatic and parallel process. We also confirmed that the judgments involved computing the mean size of an array by showing that they were almost as accurate when the distributions differed as when they were the same.

In the present research, we used dual-task paradigms to test the idea that distributed attention facilitates statistical processing. We asked participants to combine 
statistical judgments of the mean size of circles with a second concurrent task that had been shown to encourage either distributed attention or focused attention to one element at a time. We contrasted two different pairs of tasks, one of each pair requiring distributed attention and one focused attention. The first pair involved visual search, contrasting a target that popped out with a target that did not. The second required the participants to judge the orientation of either a large or a small rectangle.

To measure the accuracy of statistical processing in the context of these concurrent tasks, we again adapted the paradigm developed by Ariely (2001). On each trial, the participants responded to one of the concurrent tasks and then decided either which of two test circles matched the mean size of the circles in the previous display or which of two test circles had been presented as an individual element in the previous array. We used a staircase procedure, varying the size difference of the two probes to achieve $80 \%$ accuracy. Our hypothesis was that distributed attention would be more effective than focused attention for extracting the mean size of the circles, but not for judging the individual sizes. The suggestion is that the mean is a statistical property of the set as a whole and that it is directly accessed as such, rather than by summing the individual sizes and dividing by their number.

In the last two experiments, we tested whether simultaneous presentation of a set is more efficient for statistical processing than successive presentation is. Statistical processing has previously been tested with successive presentation. For example, Helson (1947) showed that the scale of weight judgments was centered on a weighted logarithmic mean of the successively presented weights. Posner and Keele (1968) presented a sequence of distortions of a particular nonsense shape (made from dots) and found that the prototypical shape came to represent the set in visual memory. In these experiments, we studied learning across trials and representations in memory. We were interested in seeing whether the perceptual processing mode that we studied with simultaneous displays could also be applied to a sequence of elements. We asked for mean judgments with eight circles presented either simultaneously or successively in different display locations.

\section{EXPERIMENT 1}

The purpose of Experiment 1 was to explore what deployment of attention would be most compatible with extracting statistical properties such as mean size. We hypothesized that statistical properties might be best represented when attention was distributed over a scene as a whole, whereas individual elements might be better discriminated with attention focused on each in turn. To control the deployment of attention, we used a dual-task paradigm in which the concurrent task would encourage either focused or distributed attention.

Search for a closed circle among circles with gaps has been found to require focused attention to each item in turn, whereas a circle with a gap among closed circles pops out of the display (Treisman \& Souther, 1985). If distributed attention facilitates extracting the mean size of a set and focused attention facilitates recognizing individual items, concurrent search for a circle with a gap should lead to better performance in discriminating the mean size, whereas concurrent search for a closed circle should lead to better performance in identifying the size of a randomly selected individual item. To check that our manipulation did control the deployment of attention, we varied the number of elements in the display between 4 and 12 . If focused attention is required to detect the closed circle, the search time should increase between displays of 4 and 12 items, whereas if distributed attention is used to detect the circle with a gap among closed circles, the number of elements should have no effect.

\section{Method}

Participants. Twelve participants, including the first author, took part in the experiment. All were students at Princeton University. All had normal or corrected-to-normal vision.

Apparatus and Stimuli. The stimuli were created with the Psychophysics Toolbox (Brainard, 1997) and were presented on the screen of an Apple 17-in. monitor, which was driven by a Macintosh G3. The participants were seated approximately $45 \mathrm{~cm}$ from the screen. At this distance, a pixel was approximately $0.03^{\circ}$ of visual angle. Each display consisted of a gray background and white outline circles or circles with gaps that deleted $12.5 \%$ of the circumference. The luminance of the circles was $49.9 \mathrm{~cd} / \mathrm{m}^{2}$, and the luminance of the gray background was $25.0 \mathrm{~cd} / \mathrm{m}^{2}$. Half the displays had a target, and half did not. In the focused attention condition, the target was a closed circle, and the nontarget elements were circles with gaps. In the distributed attention condition, the target was a circle with a gap, and the nontargets were closed circles. The locations of the gap centers were randomly chosen among four positions $\left(80^{\circ}, 170^{\circ}, 260^{\circ}\right.$, and $\left.350^{\circ}\right)$.

The circles in both conditions were of four different sizes, equally spaced on a $\log$ scale separated by a factor of 1.15 . The mean circle diameter was $3^{\circ}$, and the diameters ranged from $2.4^{\circ}$ to $3.7^{\circ}$. Sets with 4 and 12 circles were used, with 1 or 3 circles in each of the four sizes. The sets with 4 and 12 circles were presented in randomly chosen locations in imaginary $3 \times 3$ and $5 \times 5$ matrices, respectively. The $5 \times 5$ matrix was centered at fixation, and the $3 \times 3$ matrix was randomly located within the $5 \times 5$ matrix. The length and width of a cell was $6^{\circ}$. An example of a display is shown in Figure 1, together with two sample test circles.

The participant's response to the visual search task was followed immediately by two test circles presented $7.5^{\circ}$ to the left and right of fixation. One of the test circles matched either the mean size of the set or the size of one member of the set. The other test circle was either slightly larger or slightly smaller in size than the first test circle. The difference in size was determined by a staircase procedure depending on the performance of the previous trials. The initial size difference was a $50 \%$ diameter difference. If the participants were correct three trials in a row, the difference decreased by $3 \%$. If they were incorrect in the previous trial, the difference increased by $3 \%$. In each trial, all of the circles shown were randomly scaled by a small multiplicative factor, to discourage the observer from basing judgments on previously seen stimuli. Four multiplicative factors $(1,1.1,1.2,1.3)$ were used, and the same factor scaled all the circles in any given trial.

Design. There were four independent variables, all of which were varied within participants. The first variable was type of visual search (target closed or open circle); the second was type of size 


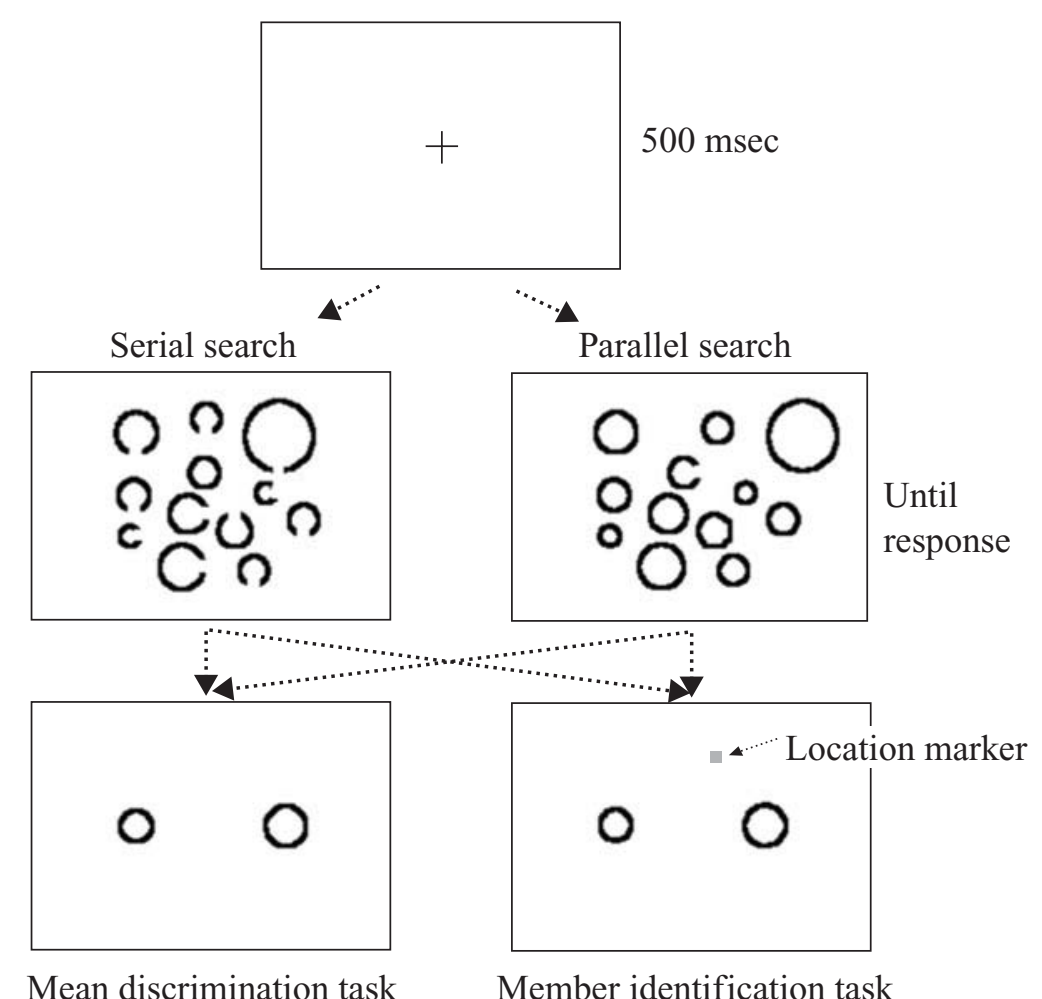

Figure 1. The stimuli and the timeline for Experiment 1. The participants first did one of two search tasks and then did one of the size judgments tasks.

judgment (either mean or member). Both of these were blocked. The third variable was target presence (either present or absent); and the fourth was set size (either 4 or 12 items). Both these factors were randomly mixed within blocks. Each participant served in four experimental blocks ( 2 types of visual search $\times 2$ types of size judgment), as well as four practice blocks. The order of the experimental blocks was counterbalanced between participants. The order of trials within each block (target present or absent $\times$ display size 4 or 12) was randomly selected, under the constraint that each condition was presented once before any condition was repeated. There were 50 trials in the practice blocks, and the experimental blocks continued until 17 reversals had occurred in the staircase procedure. The initial diameter difference in the experimental block was the last diameter difference in the practice block.

Procedure. A timeline of the procedure is shown in Figure 1. Each trial started with a fixation cross for $500 \mathrm{msec}$, followed by a search display that remained present until the participant responded. The participants' first task was to search for either an open or a closed circle (the target). They pressed 1 when the target was present and 2 when it was not. When their decision was incorrect, they heard a brief high tone.

After they had responded to the search task, two circles appeared in the center of the screen. The second task was to decide which of the two test circles matched the mean size of the circles in the previous display or which of the two test circles had been presented as an individual element at the location indicated by a marker on the screen. The marker was a small white square, which appeared at the same time as the two test circles. In the member identification task when the target was present, the target was always presented as one of the two test circles. The participants pressed 1 when they thought that the left circle was the answer and 2 when they thought that the right circle was the answer. They received feedback, as they had in the first task.

We used a staircase procedure to determine the size judgment threshold, varying the diameter difference of the two test circles to achieve $80 \%$ accuracy. The step size was a $3 \%$ diameter difference between the two test circles. We used the mean of the last 12 reversals as the threshold for size discrimination.

\section{Results and Discussion}

The results of Experiment 1 are shown in Figure 2. Figure 2A shows the results of the visual search. The participants were faster when they searched for a pop-out target (a circle with a gap) than when they searched for a non-pop-out target [a closed circle; $F(1,11)=60.4$, $p<.01]$, and they were faster when the set size was 4 than when the set size was $12[F(1,11)=114.9, p<$ $.01]$. The interaction between type of visual search and set size was significant $[F(2,32)=72.3, p<.01]$. The mean slope relating search times to set size was relatively shallow for the pop-out target (averaging $14 \mathrm{msec} /$ item for target present and $35 \mathrm{msec} /$ item for target absent). Unlike the results of Treisman and Souther (1985), the target-absent slope was significant $[t(11)=4.7, p<$ $.01]$, suggesting that there may have been some interference from the competing dual task. However, the slope for the non-pop-out target was much steeper [71 msec/item for target present, $t(11)=9.8, p<.01 ; 181 \mathrm{msec} /$ item for target absent, $t(11)=11.2, p<.01]$. The participants 

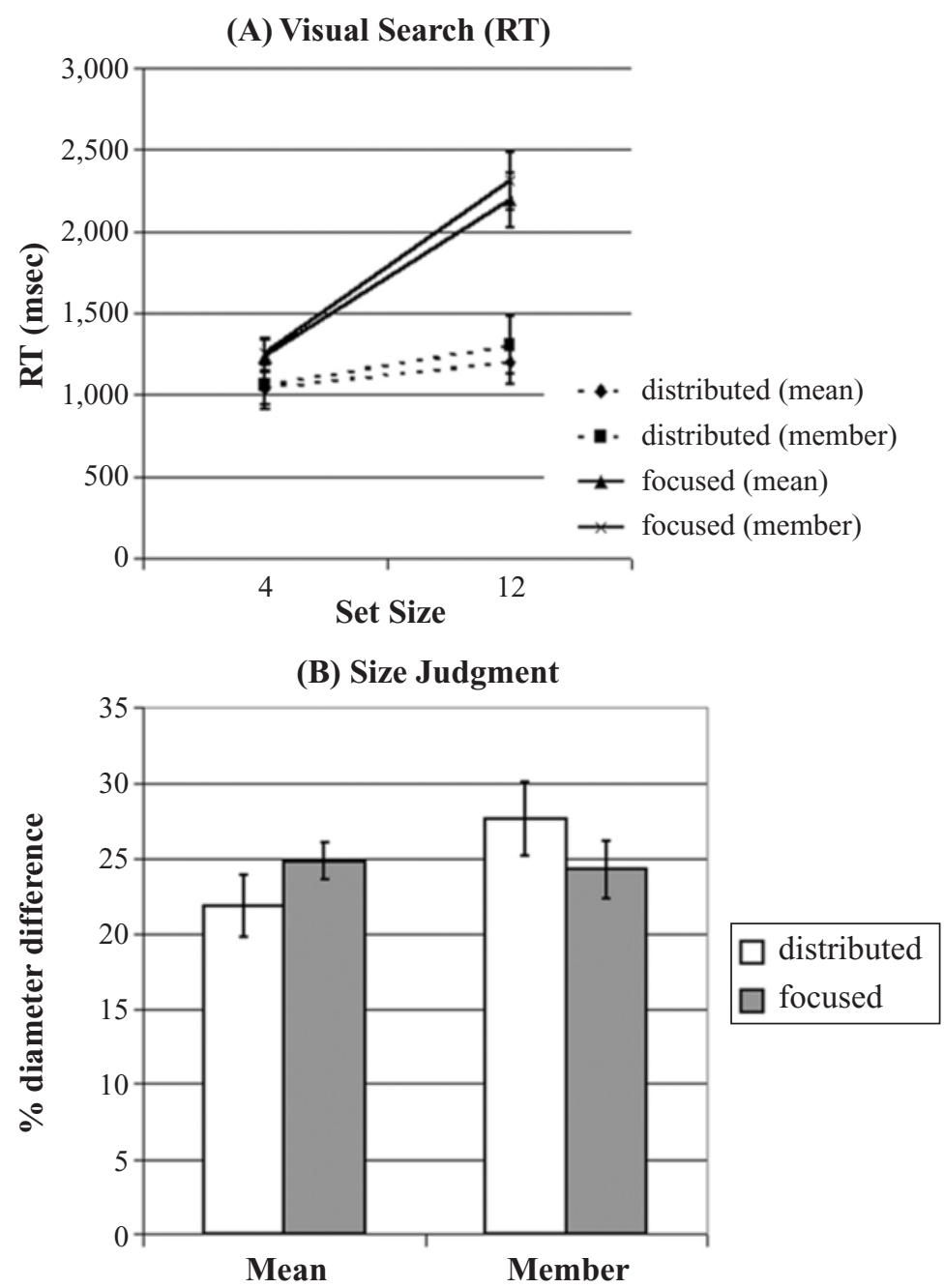

Figure 2. The results of Experiment 1. The error bars indicate standard errors. (A) Distributed indicates searching for a circle with a gap among circles without a gap, and focused indicates searching for a circle without a gap among circles with a gap. (B) Mean indicates a mean discrimination task, and member indicates a member identification task.

may have attended to groups of items in searching for a $\mathrm{C}$ and to individual items in searching for an $\mathrm{O}$. No other two-way or three-way interactions were significant.

The error rates in the search task with focused attention were $7 \%$ and $6 \%$ for the concurrent mean and member judgments, respectively; with distributed attention, the corresponding rates were $2 \%$ and $3 \%$. An analysis of variance showed that the participants were more accurate when they searched for a pop-out target than when they searched for a non-pop-out target $[F(1,11)=26.2$, $p<.01]$. Accuracy in visual search was not affected by which of the two size judgment tasks it accompanied (mean or member, $p=.78$ ), and the two-way interaction was not significant.

Figure 2B shows the results of the size judgment task. Although neither comparison between the two attention deployments quite reached significance within the two size judgment tasks $[t(11)=1.5, p=.15$, for the mean size judgment; $t(11)=1.8, p=.1$, for the member size judgment], the interaction of size judgment task and attention deployment was significant, as was predicted $[F(1,11)=9.6, p<.05]$. In the mean size judgment, the thresholds were $22 \%$ with the $\mathrm{C}$ target and $25 \%$ with the $\mathrm{O}$ target, whereas in the member size judgments, they were $28 \%$ with the $\mathrm{C}$ target and $24 \%$ with the $\mathrm{O}$ target.

In the member identification task when the target was present, the target was always presented as one of the two test circles. In order to see whether size judgments differed between target-present and target-absent trials, we reanalyzed the member identification performance depending on the presence of the target, using percentage correct, rather than thresholds. Member size judgments were better on target-present than on target-absent trials $[t(11)=4.6, p<.01$, mean $=89 \%$ vs. $78 \%$ with the 
pop-out search; $t(11)=6.9, p<.01$, mean $=92 \%$ vs. $74 \%$ with the serial search]. In both search tasks, the participants presumably attended to the target immediately before responding. Memory for the size clearly benefited from this recent attention.

The critical question that we tested in Experiment 1 was whether the type of search task would differentially affect the two types of size judgment. We predicted that judgments of mean size would benefit more from the pop-out search condition because it induced more distributed attention. Indeed, we found the predicted interaction between the type of search task and the type of size judgment. Could some other factor have caused the interaction? The relevant discrimination in the search tasks was based on the same feature (a gap in the circles), which bears no obvious relation to size differences. Difficulty was approximately equated by leaving the display up until the participant responded, resulting in much longer durations with the $\mathrm{O}$ targets. The tasks required the participants to search as fast as possible, using whatever capacity was available. Thus, it is likely that the deployment of attention was the critical parameter distinguishing the two conditions. Note that search was not completely parallel in the pop-out condition, perhaps because there was some interference from the need to coordinate the two tasks. The interaction between type of size judgment and search condition might have been even stronger if attention had been parallel across the whole display.

\section{EXPERIMENT 2}

The concurrent tasks in Experiment 1 contrasted focused and distributed attention. To generalize the results, in Experiment 2, we used a different concurrent task to modulate the deployment of attention, requiring the participants to judge the orientation of either a large rectangular frame around the display or a small rectangle at the center, while again judging the mean size of the circles in the display. The concurrent tasks differed from those in Experiment 1 by requiring attention to different perceptual units, other than the circles on which the size judgments were to be made. The participants were to say whether a rectangle was horizontally or vertically oriented. The rectangle was either a large one surrounding the circles or a small one at the fovea. Thus, the tasks required that attention be divided between different stimuli (rectangles and circles), as well as between different tasks (orientation judgment and size judgment). Our hypothesis was that attention to the large rectangle would be easier to combine with distributed attention to the circles than would attention to the small rectangle.

\section{Method}

Participants. Ten Princeton undergraduate students participated in the experiment for the option of course credit. All had normal or corrected-to-normal vision.

Apparatus and Stimuli. The apparatus in Experiment 2 was the same as that in Experiment 1. The displays were the same as those in Experiment 1, with the following exceptions. All the circles were closed, and there was no target. The sizes were equally spaced on a $\log$ scale separated by a factor of 1.15 . The mean circle diameter was $1.25^{\circ}$, and the diameters ranged from $1^{\circ}$ to $1.52^{\circ}$. The participants were seated approximately $79 \mathrm{~cm}$ from the screen. All the displays consisted of 16 circles, presented in an imaginary $5 \times 5$ matrix located in the center of the screen. The length and width of a cell was $3^{\circ}$. The positions of the circles were randomly chosen among the matrix cells, except the cell in the center. The method of choosing test circles was the same that as in Experiment 1, as was the luminance of both the stimuli and the background.

A small rectangle was always presented in the center cell, and a large rectangle surrounded the display of circles. An example of a display is shown in Figure 3. The initial size of the large rectangle was either $17.8^{\circ} \times 15.1^{\circ}$ or $15.1^{\circ} \times 17.8^{\circ}$, and the initial size of the small rectangle was either $1.2^{\circ} \times 1.02^{\circ}$ or $1.02^{\circ} \times 1.2^{\circ}$. Thus, the aspect ratios were matched. To equate the difficulty of the orientation judgment between the large and the small rectangles, the aspect ratio was determined by a staircase procedure depending on the participant's performance in the previous trials. A single staircase was used for the attended rectangle. A step size was a change of $1 \%$ in the aspect ratio. If the participants were correct three trials in a row, the difference decreased by $1 \%$. If they were incorrect in the previous trial, the difference increased by $1 \%$. The aspect ratio was varied between $6 \%$ and $17 \%$.

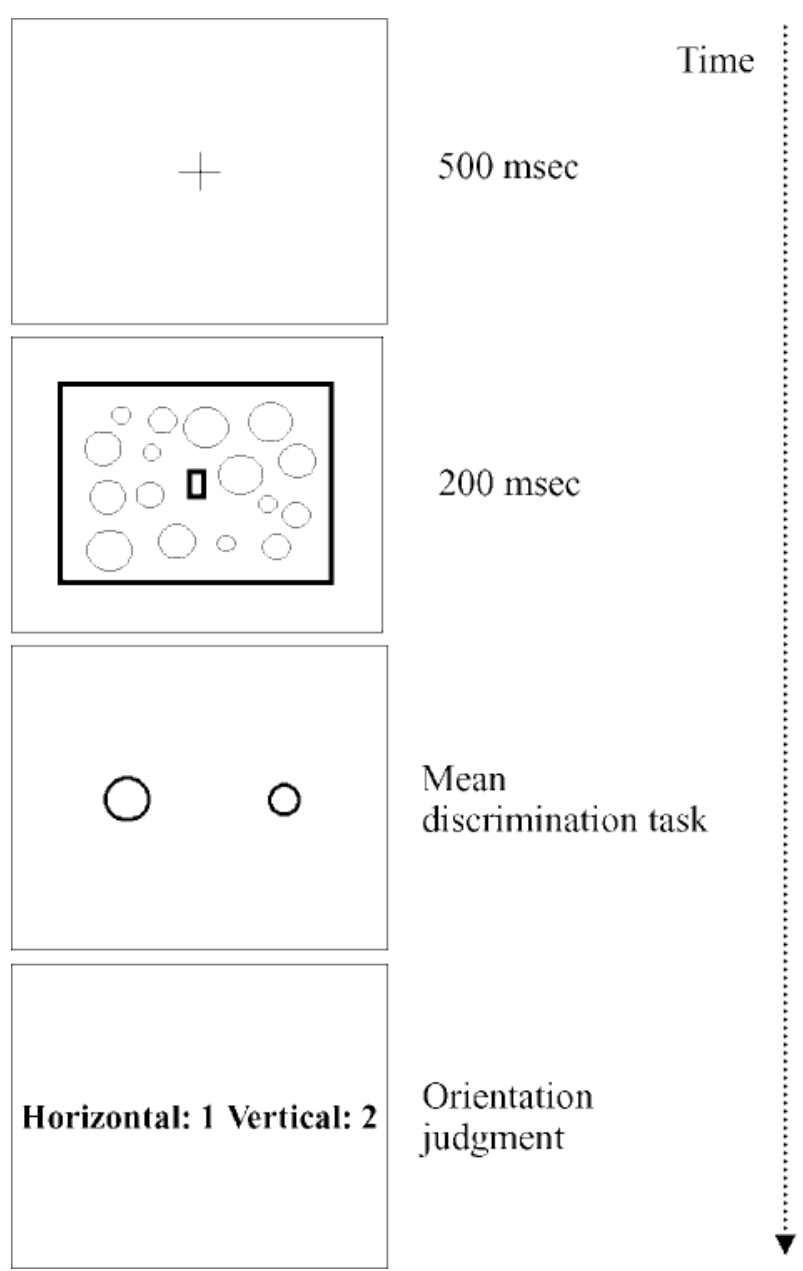

Figure 3. The stimuli and the timeline for Experiment 2. The participants first did the mean discrimination task and then did one of the orientation judgment tasks. 
Design. In this experiment, only the mean size judgment was tested in addition to the orientation judgment, so there was just one independent variable, either global or local attention. This was varied within participants by selecting whether the concurrent task was to judge the orientation of the large or the small rectangle. Each participant served in four blocks (two for each type of attention), as well as four practice blocks. The practice blocks had 50 trials, and the experimental blocks ended after 13 reversals of the staircase. The two attention conditions were tested in separate blocks, with their order counterbalanced across and within participants.

The staircase procedure for the size judgment was the same as that in Experiment 1, except that we collected 13 reversals, rather than 17 reversals, during each of the two experimental blocks. We used the average of the two means of the last 12 reversals as the threshold of size discrimination.

Procedure. A timeline of the procedure is shown in Figure 3. Each trial started with a fixation cross for $500 \mathrm{msec}$, followed by a display $(200 \mathrm{msec})$, which in turn was followed by the two test circles. Unlike in Experiment 1, in which the search task preceded the mean size judgment, the participants' first task here was to decide which of the two test circles matched the mean size of the circles in the previous display. They pressed 1 when they thought that the left circle was the answer and 2 when they thought that the right circle was the answer. When their decision was incorrect, they heard a brief high tone. After they had responded to the size task, key instructions for the orientation task appeared. A relevant rectangle was designated at the beginning of each block, and the relevant rectangle was varied across blocks. The participants pressed 1 when the designated rectangle was aligned horizontally and 2 when it was aligned vertically. A constant reminder of key mapping was always present on the screen. The participants received feedback, as they had in the first task.

\section{Results and Discussion}

The results of Experiment 2 are shown in Figure 4. Figure 4A indicates the results of the orientation judgment task. There was no significant difference in accuracy between the two orientation judgments $[t(9)=1.2$, $p=.25]$, suggesting that performance on the global and the local orientation judgments was closely matched in difficulty, as we had hoped.

Figure 4B shows the results of the size judgment task. Thresholds for the mean size were lower when the large rectangle was relevant than when the small rectangle was relevant in the concurrent orientation task, $[t(9)=2.7$, $p<.05]$. We found the same effect in a pilot experiment with a set size of 4 rather than a set size of $16[t(7)=$ $-3.2, p<.05]$. There were 8 participants in the pilot experiment, and the method was identical, except for set size. Thus, we found the predicted advantage of global attention for judging the mean size of an array. In both conditions in this experiment, attention was directed to a rectangle, as well as to the circles. However, discriminating the orientation of the large rectangle required that attention be spread across the display, allowing easier concurrent processing of the array as a whole, which in turn seems to have favored easier extraction of its statistical properties. The fact that the scale was different for the large rectangle does not seem to have prevented statistical processing of the circles to determine their mean size.

In order to see whether numerosity affects the accuracy of statistical processing, we compared the pilot ex- periment (set size of 4) and this experiment (set size of 16). The threshold difference in size judgments between global and local attention did not differ significantly across the two experiments $[t(16)=1.35, p=.20]$. The overall thresholds did not differ significantly across the two experiments $[t(34)=2.0, p=.053]$, nor did the thresholds with global attention $[t(16)=1.3, p=.21]$ or the thresholds with local attention $[t(16)=1.5, p=.16]$. This result is consistent with Ariely's finding (2001) that there was no set size effect when the mean size of a set was judged.

Thresholds were lower in Experiment 1 than in Experiment 2 , averaging $25 \%$ versus $37 \%[t(20)=2.3, p<.05]$ with focused attention and $22 \%$ versus $31 \%[t(20)=2.1$, $p<.05]$ with distributed or global attention. There are several possible reasons for this difference. First, the concurrent tasks were different: The orientation judgment could be more demanding than the search task. Second, the concurrent tasks in Experiment 2 required attention to different elements (the rectangle and the circles), whereas in Experiment 1 attention was paid to different properties of the same stimuli (the shape and size of the circles). We know that attention is more easily divided between attributes of the same objects than between different objects (Duncan, 1984; Treisman, 1969). Experiment 2 also required two different forms of attention deployment - global to the large-scale rectangle and distributed to the local, small-scale circles - whereas Experiment 1 required distributed attention to the local circles for both tasks in the pop-out condition. Further research is needed to clarify which difference is responsible for the difference in statistical thresholds.

\section{EXPERIMENT 3}

In both experiments, focused attention proved less compatible than global or distributed attention when the mean size of the circles was judged. This is consistent with the existence of a special perceptual mode of statistical processing that requires parallel attention to a set of simultaneously presented stimuli. On the other hand, previous research has shown that participants can also form a memory representation of the mean when stimuli are presented successively over longer intervals (Helson, 1947).

One possible mechanism that could underlie the statistical judgments of size in our experiments is the neural averaging of responses in single units to visual stimuli within their receptive fields observed by Desimone and Duncan (1995; Reynolds, Chelazzi, \& Desimone, 1999) and the presumably related reduction of fMRI responses in human participants when stimuli are presented simultaneously rather than successively (Kastner, De Weerd, Desimone, \& Ungerleider, 1998). These authors related their observations to the biased competition account of attention and attributed the reduced response to mutual inhibition between object representations. It does, however, have the effect of averaging the responses to simultaneously presented stimuli and could play a role in 
(A) Orientation Judgment

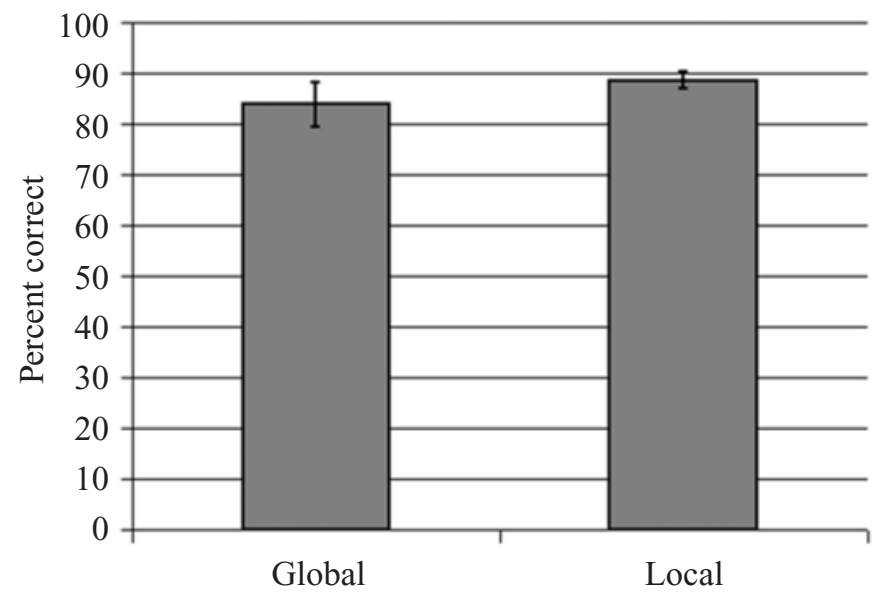

(B) Size Judgment

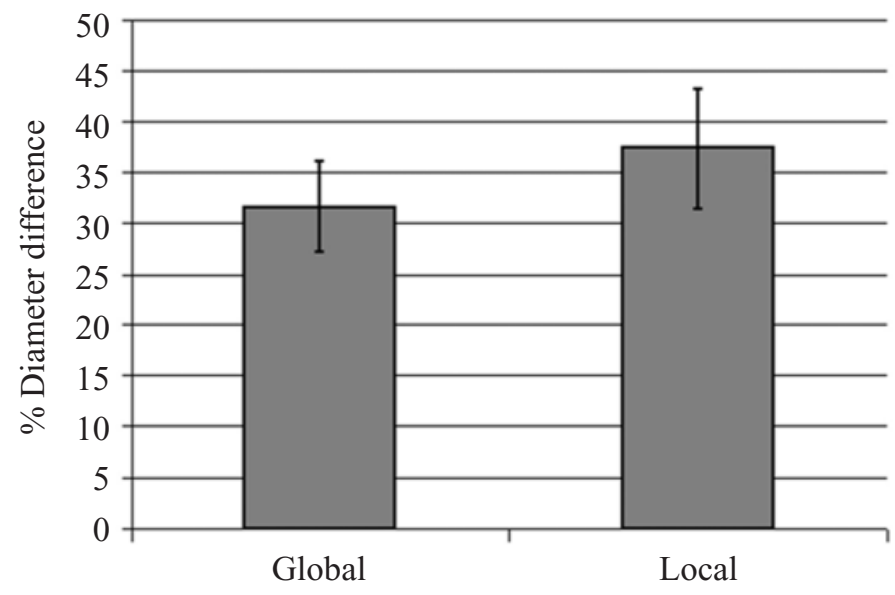

Figure 4. The results of Experiment 2. Global indicates deciding the orientation of the large rectangle surrounding circles, and local indicates deciding the orientation of the small rectangle at the center of the screen.

registering the statistical mean in our experiments so far. One way to test this account would be to compare simultaneous with successive presentation of the stimuli to be averaged. Experiment 3 presented the same stimuli either simultaneously or successively, to compare the efficiency of statistical processing in the parallel mode and in the successive memory integration mode.

\section{Method}

Participants. Ten Princeton undergraduate students participated in the experiment for the option of course credit. All had normal or corrected-to-normal vision.

Apparatus and Stimuli. The apparatus for Experiment 3 was the same as that in Experiment 1. Each display consisted of white outline circles. An example of a display is shown in Figure 5. The sizes were equally spaced on a power function with an exponent of 0.76 (the psychological scale for size; Teghtsoonian, 1965). Since the participants were seated approximately $79 \mathrm{~cm}$ from the screen, a pixel was approximately $0.02^{\circ}$ of visual angle. The mean circle diameter was $1.4^{\circ}$, and the diameters ranged from $1^{\circ}$ to $1.7^{\circ}$. Sets with eight circles were used, comprising two from each of four sizes. The circles in both the simultaneous and the successive conditions were presented in randomly chosen positions in an imaginary $3 \times$ 3 matrix located in the center of the screen. In each trial, the circles were randomly scaled by a small multiplicative factor, to discourage the observers from basing their judgments on previously seen stimuli. Four multiplicative factors $(1,1.1,1.2$, and 1.3) were used, and the same factor scaled all circles in any one trial. The method of choosing test circles and the luminance of both the stimuli and the background were the same as those in Experiment 1.

Design. There were two independent variables in the experiment, both of which were varied within participants. The first variable was type of size judgment task - either mean or member identification. These were presented in separate blocks, counterbalanced across subjects. The second was type of presentation - either successive or simultaneous. With simultaneous presentations, two durations were tested, $250 \mathrm{msec}$ and $2 \mathrm{sec}$. These three types of presentation were randomly mixed within blocks, under the constraint that each condition was presented once before any condition was repeated.

Each participant served in two blocks (two types of task), as well as in two practice blocks. The practice blocks ended when 4 rever- 

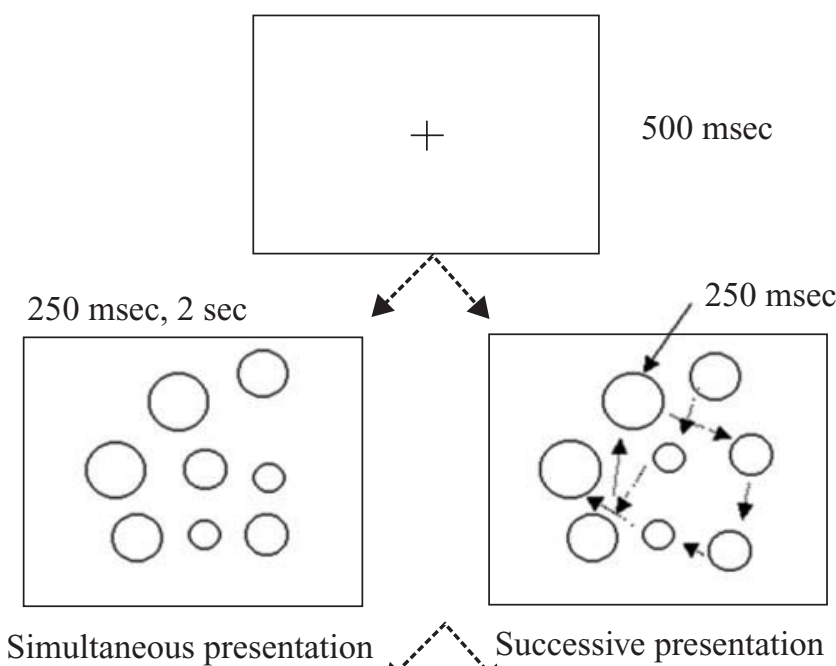

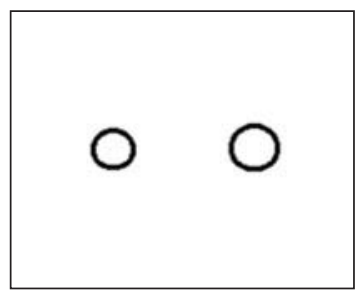

Mean discrimination task

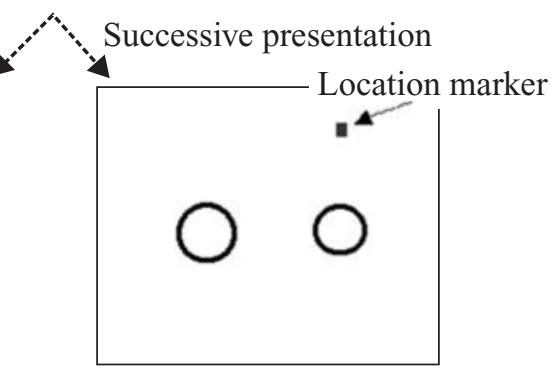

Member identification task

Figure 5. The stimuli and the timeline for Experiment 3. In the simultaneous presentation, there were two different durations $(250 \mathrm{msec}$ and $2 \mathrm{sec})$. In the successive presentation, each item was presented at a randomly chosen position for $250 \mathrm{msec}$, and it never appeared at the same location.

sals of the staircase procedure had occurred, and the experimental blocks ended after 13 reversals. The staircase procedure for the dependent measurement was the same as that in Experiment 1, except that we collected 13 reversals, rather than 17 reversals, during each experimental block.

Procedure. A timeline of the procedure is shown in Figure 5. Each trial started with a fixation cross for $500 \mathrm{msec}$, followed by a display. The duration of the display was $250 \mathrm{msec}$ in the short simultaneous presentation and $2 \mathrm{sec}$ in the long simultaneous presentation. It was $250 \mathrm{msec}$ for each circle in the successive presentation, making a total of $2 \mathrm{sec}$. After the display, two circles appeared in the center of screen. The participants' task was to decide which of the two test circles matched the mean size of the circles in the previous display or which of the two test circles had been presented as an individual element at the location indicated by a marker on the screen. The marker was a white square that appeared at the same time as the two test circles. The participants pressed 1 when they thought that the left circle was the answer and 2 when they thought that the right circle was the answer. When their decision was incorrect, they heard a brief high tone.

\section{Results and Discussion}

The results of Experiment 3 are shown in Figure 6. Thresholds were lower for the mean identification task than for the member identification task $[F(1,9)=259.177$, $p<.01]$. There were significant threshold differences depending on presentation types $[F(2,18)=6.245, p<$ $.01]$. $t$ tests showed that the long simultaneous presenta- tion gave a significantly lower threshold than did short simultaneous presentation, both for the mean identification task $[t(9)=2.316, p<.05]$ and for the member identification task $[t(9)=2.744, p<.05]$. The successive presentation condition did not differ significantly from either of the simultaneous conditions. The interaction between type of task and type of presentation was not significant $[F(2,18)=0.415, p=.67]$.

These results seem inconsistent with the hypothesis that extracting statistical descriptors is more efficient with parallel access to the array than with serial access. They show that the participants can also extract the mean by accumulating representations of successive items and that this may be as efficient as processing a simultaneous display. The small (3\%) benefit from the longer exposure with simultaneous presentation suggests that statistical processing is not instantaneous. This benefit is comparable to the finding by Chong and Treisman (2003) that mean discrimination thresholds decreased by $2 \%$ when the duration of the stimulus increased from $50 \mathrm{msec}$ to $1 \mathrm{sec}$. However, the three presentation modes may have given similar results simply because the four different sizes appeared equally often in each trial, allowing the participants to attend to just one of each. This might have been a particularly attractive strategy with successive 


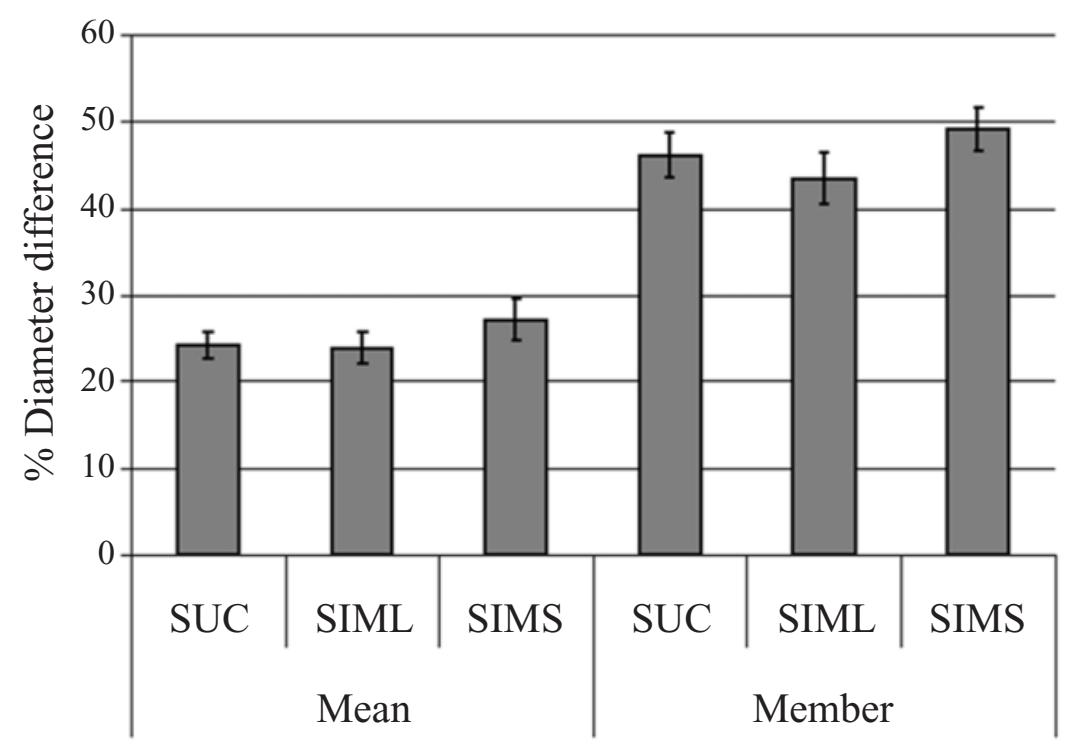

Figure 6. The results of Experiment 3. SUC indicates the successive presentation, SIML stands for the long simultaneous presentation, and SIMS stands for the short simultaneous presentation.

presentation, where full attention could be devoted to each circle, making the structure of the sequence clearly apparent. We tested this possibility in Experiment 4.

\section{EXPERIMENT 4}

In this experiment, we used only two sizes but varied their relative frequency in different displays, making it impossible to store just one instance of each size. By varying the frequencies of each size, we ensured that the whole set would have to be processed to ensure accurate judgments.

\section{Method}

Participants. Eight Princeton undergraduate students and 2 experienced participants (including the first author) served in the experiment. All had normal or corrected-to-normal vision. The 8 undergraduate students participated for the option of course credit.

Apparatus and Stimuli. The apparatus, the stimuli, and the luminance were the same as those in Experiment 3, with the following exceptions: Only the smallest and the largest sizes were used in five different frequency distributions - two instances of the smallest with six instances of the largest circle, three instances of the smallest with five instances of the largest circle, four instances of each of the two sizes, five instances of the smallest with three instances of the largest circle, and six instances of the smallest with two instances of the largest circle.

Design and Procedure. The design of Experiment 4 was the same as that in Experiment 3, with the following exceptions: The member identification task was not included, and instead, the participants did the mean identification task twice. Consequently, the threshold was defined as the average of the two means of the last 12 reversals.

The 2 experienced participants did the whole experiment one additional time with the positions of the eight circles fixed at the center of the screen (with a small jitter to prevent identical circles from exactly overlapping each other), instead of appearing successively in a series of different locations. We compared performance in this fixed location condition with performance in the successive presentation conditions in which the circles were presented in different locations in a randomized order.

\section{Results and Discussion}

The results of Experiment 4 are shown in Figure 7. There were significant threshold differences depending on presentation types $[F(2,18)=7.33, p<.01] . t$ tests showed that the long simultaneous presentation gave significantly lower thresholds than both the short simultaneous presentation $[t(9)=3.355, p<.01]$ and the successive presentation $[t(9)=4.684, p<.01]$. The thresholds in the short simultaneous presentation did not differ from those in the successive presentation $[t(9)=1.432, p=.19]$.

The average threshold for the 2 experienced participants in the successive presentation with fixed position $(20 \%)$ was exactly the same as that with the random positions $(20 \%)$, suggesting that the random versus fixed positions in the successive presentation made no difference to accuracy in extracting the mean size.

Given the same total exposure time, the results show that the mean size can be extracted somewhat more accurately with simultaneous displays than through the accumulation across time of serially presented items. These results are consistent with the hypothesis that extracting statistical descriptors benefits from parallel access to all the information in an array. However the difference is quite small, and the simultaneous brief exposure is only slightly worse, despite the eightfold reduction in exposure time. The thresholds are also very similar to those in Experiment 3, suggesting that the participants were extracting the mean in both cases, rather than using a few exemplars when the frequencies were matched. 


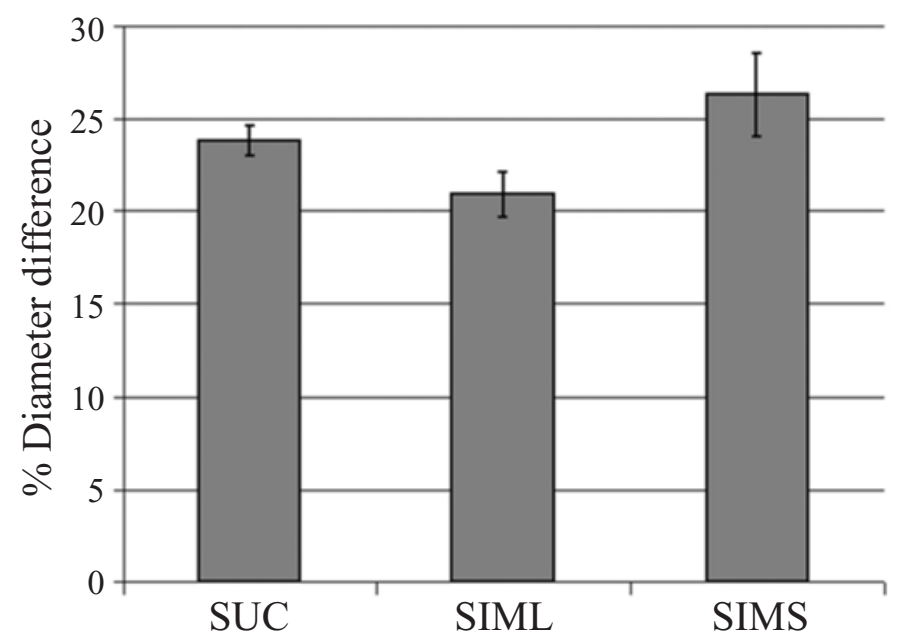

Figure 7. The results of Experiment 4. SUC, successive presentation; SIML, long simultaneous presentation; SIMS, short simultaneous presentation.

The comparison of simultaneous with successive presentations is interesting in the context of brain-imaging experiments by Kastner et al. (1998) in which successive and simultaneous presentations were compared and the studies of single units by Reynolds et al. (1999). The biased competition model of attention (Desimone \& Duncan, 1995) suggests that stimuli reaching the same receptive field are mutually inhibitory, as if competing for representation. The observations are equivalent to a form of averaging, rather than summing of activation within receptive fields, which suggests the possibility that our statistical-averaging data reflect the same neural mechanism. If this were the case, however, we might expect a more dramatic change when we switch from simultaneous to successive presentation.

\section{GENERAL DISCUSSION}

The term attention is used in two different senses, both of which could be relevant to statistical processing in perception: (1) Attention can refer to the selection of items in a particular spatial area for enhanced processing, and (2) it can refer to the allocation of resources from a limited capacity pool to one task at the expense of another. The research described here may throw some light on both. The hypotheses we tested were (1) that statistical processing is a distinct mode of perceptual analysis that accompanies distributed attention to sets of similar objects and (2) that given this distributed deployment of attention, statistical processing is automatic, occurring without additional effort or resources.

\section{Spatial Attention and Statistical Processing}

The first two experiments used a dual-task paradigm to vary the spatial deployment of attention. Either statistical judgments of the mean size or judgments of the size of specific individual items were made while the participants also worked on a concurrent task designed to en- courage attention either to the array as a whole or to local elements. The prediction was that performance would be better on judgments of mean size when the concurrent task encouraged distributed or global attention and on judgments of individual size when the concurrent task required focused attention to each element in turn. The results generally supported the hypothesis. We found the predicted interaction in both of the experiments.

In Experiment 1, the participants searched either for a target that popped out when attention was distributed over the set as a whole ( $\mathrm{C}$ among $\mathrm{Os}$ ) or for a target that required focused attention to each item in turn (O among $\mathrm{Cs})$. The threshold for judging the mean size was lower with the pop-out target, even though the exposure duration was only half as long. (The exposure was terminated when the participant responded, and search times were considerably slower with the $\mathrm{O}$ target among $\mathrm{Cs}$ ). If anything, the reverse was the case when size judgments were required on an individual item; here, thresholds were lower with focused attention (search for $\mathrm{O}$ among Cs). Size judgments on individual items presumably require the relevant circle to be individuated, which we believe needs focused attention. Consistent with this hypothesis, thresholds were also lower in target-present than in target-absent trials. When the target was present, the probed size was always that of the target. Presumably, this received more attention than the distractors, although the total exposure time was longer on targetabsent trials.

In Experiment 2, orientation judgments were used as the concurrent task, and the scale of the shape to be judged was varied. Local attention was induced by getting participants to judge the orientation of a small local rectangle in the center of the display, whereas global attention was induced by asking for the orientation of a large rectangle that framed the display. The threshold for the mean size was lower when the concurrent orientation judgment was made on the global shape. 
Searching for a $\mathrm{C}$ among Os in Experiment 1 required distributed attention to the same display elements at the same scale as that for the size judgments, whereas judging the orientation of the large rectangle in Experiment 2 required global attention to a different display element at a different scale. Searching for an $\mathrm{O}$ among Cs in Experiment 1 required focused attention to the same elements as those for the size judgments, whereas judging the orientation of a center rectangle in Experiment 2 required local attention to a local element different from the size stimuli. These contrasts might contribute to the explanation of threshold differences between the two experiments. Thresholds were lower in Experiment 1 (22\% and $25 \%$ for distributed and focused attention, respectively) than in Experiment 2 (31\% and 37\% for global and local attention, respectively). The advantage in Experiment 1 was obtained despite the fact that the size judgments were made after the response to the search task, whereas in Experiment 2 they were made before the orientation judgments. This increases the weight of the evidence that distributed attention to the same elements is more efficient for statistical processing than is global attention to a surrounding element. However, these results cannot be taken as conclusive evidence for the effects of different deployments of attention, since the tasks were also different. It could be the case that there is greater competition between orientation and size judgments than between search and size judgments. Further research will be needed to sort out the contributions of these different factors. The difference could also be due to attentional transition time from the large or the small rectangle to the circles in Experiment 2.

We tested a further prediction derived from the idea that statistical properties become more easily available through parallel processing of global sets: If this were the case, simultaneous exposure to a whole set of items might yield more efficient extraction of statistical properties. We compared judgments of the mean size when the items were presented sequentially, one at a time, and when the whole display was available at once. Experiment 3 showed no difference in accuracy with simultaneous and with successive presentation but may have allowed the participants to base their responses on just a subset of items. Experiment 4 blocked this strategy and forced the participants to respond more specifically to the mean size. Here, we did find an advantage for simultaneous over successive presentation when the total time available was matched. However, the difference was small, and the simultaneous presentation with a short exposure showed no benefit over successive presentation. This makes it unlikely that the kind of neural averaging observed in single units and fMRI is mediating our results.

\section{Automaticity of Statistical Processing?}

Comparisons across the four experiments can throw some light on the second question that interested uswhether the size is registered automatically or depends on limited capacity resources. Experiments 1 and 2 and the pilot experiment measured thresholds for judging the mean while carrying out a concurrent task, whereas Experiments 3 and 4 tested the mean identification task on its own The same method being used across all four experiments. The mean identification thresholds $(27.7 \%)$ from the dualtasks were significantly higher overall than those $(24.4 \%)$ from the single tasks $[t(118)=2.01, p<.05]$. However, if we take only the conditions relevant to the predictionthose in which the concurrent task required distributed or global attention and in which the circles were presented simultaneously - the thresholds did not differ $[25.9 \%$ vs. $23.7 \% ; t(58)=0.986, p=.33$. Thus, provided that attention is distributed over the whole display, extraction of the mean appears not to require any additional resources. This finding is suggestive rather than conclusive, and further research comparing dual- with single-task performance within participants will be needed to confirm our hypothesis. But the fact that it applies across two different competing tasks and two single-task experiments suggests that there is unlikely to be much, if any, interference with statistical processing from a concurrent task that requires the same global distribution of attention.

Converging evidence for the automaticity of statistical judgments comes also from earlier findings. Ariely (2001) found that there was no set size effect in judging the mean size of a set. Consistent with this finding, the mean discrimination thresholds were the same for a set size of 16 as for a set size of 4 in Experiment 2. Chong and Treisman (2003) showed that statistical estimates of the mean sizes of sets of similar objects are little affected by exposure duration or delay.

\section{Comparison With Earlier Findings}

The mean size thresholds without a concurrent task reported by Chong and Treisman (2003) averaged about $5 \%-10 \%$, whereas in the present experiments they were around $25 \%$. When we compare the thresholds for the same 2 participants (including the first author) in both sets of experiments, the threshold was still considerably lower in the earlier experiments (by 12\%), suggesting that it is most likely due to differences in methods between the studies. One important difference is that the earlier experiments measured relative thresholds by asking which of the two displays had the larger mean size, whereas the present tests measured absolute thresholds by asking which of the two test circles was the mean size of the previous display. Another difference is that Chong and Treisman used the method of constant stimuli and defined the thresholds as the diameter difference that gave $75 \%$ accuracy, whereas in the present experiments, we used a staircase method and aimed for $80 \%$ accuracy. Woods and Thomson (1993) found that the method of constant stimuli generated lower thresholds than the staircase method even under the same experimental conditions.

Judgments of individual size in our experiments were generally less accurate than statistical judgments of mean size, confirming the earlier finding by Ariely (2001; Ariely \& Burbeck, 1995). Our method of measuring the 
threshold for individual items differed from Ariely's. He picked a random item to test, without indicating to the participant which one, whereas we used a location cue to indicate which item was being probed. We wanted to force the participants to focus on individual items. Our method decreases the processing load in one way, but it may have increased it in another by requiring memory for the locations. Ariely did not specify the location of the test item and may, therefore, have tested recognition of types, rather than of individual tokens.

In conclusion, we showed that it is easier to recognize the mean size of an array when a concurrent task requires distributed or global attention than when it requires focused or local attention. There may be a separate visual mechanism, enabled by a distributed or global deployment of attention, that directly extracts statistical properties without separately identifying each individual item.

\section{REFERENCES}

ARIELy, D. (2001). Seeing sets: Representation by statistical properties. Psychological Science, 12, 157-162.

ARIELY, D., \& BURBECK, C. A. (1995). Statistical encoding of multiple stimuli: A theory of distributed representation [Abstract]. Investigative Ophthalmology \& Visual Science, 36(Suppl.), No. 8472.

Austin, E., \& EnNs, J. T. (2000). Change detection: Paying attention to detail. PSYCHE, 6, 1-16.

BrainarD, D. H. (1997). The Psychophysics Toolbox. Spatial Vision, 10, 433-436.

CHONG, S. C., \& Treisman, A. (2003). Representation of statistical properties. Vision Research, 43, 393-404.

DeLis, D. C., RoberTSON, L. C., \& EFrON, R. (1986). Hemispheric specialization of memory for visual hierarchical stimuli. Neuropsychologia, 24, 205-214.

DESIMONE, R., \& DunCAN, J. (1995). Neural mechanisms of selective visual attention. Annual Review of Neuroscience, 18, 193-222.

DunCAN, J. (1984). Selective attention and the organization of visual information. Journal of Experimental Psychology: General, 113, 501517.

Farell, B., \& Pelli, D. G. (1993). Can we attend to large and small at the same time? Vision Research, 33, 2757-2772.

FIELD, D. J. (1987). Relations between the statistics of natural images and the response properties of cortical cells. Journal of the Optical Society of America A, 4, 2379-2394.

Fink, G. R., Halligan, P. W., Marshall, J. C., Frith, C. D., FrackOWIAK, R. S. J., \& Dolan, R. J. (1996). Where in the brain does visual attention select the forest and the trees? Nature, 382, 626-628.

Geisler, W. S., Perry, J. S., Super, B. J., \& Gallogly, D. P. (2001). Edge co-occurrence in natural images predicts contour grouping performance. Vision Research, 41, 711-724.

Haralick, R. M., Shanmugam, K., \& Dinstein, I. (1973). Textural features for image classification. IEEE Transactions on Systems, Man, \& Cybernetics, 3, 610-621.

Heinze, H. J., Hinrichs, H., Scholz, M., Burchert, W., \& Mangun, G. R. (1998). Neural mechanisms of global and local processing: A combined PET and ERP study. Journal of Cognitive Neuroscience, 10, 485-498.

HELSON, H. (1947). Adaptation-level as frame of reference for prediction of psychophysical data. American Journal of Psychology, 60, 1-29.
Hock, H. S., PArk, C. L., \& SchöNER, G. (2002). Self-organized pattern formation: Experimental dissection of motion detection and motion integration by variation of attentional spread. Vision Research, 42, 991-1003.

HOFFMAN, J. E. (1980). Interaction between global and local levels of a form. Journal of Experimental Psychology: Human Perception \& Performance, 6, 222-234.

JuLESZ, B. (1981). Textons, the elements of texture perception, and their interactions. Nature, 290, 91-97.

Kastner, S., De Weerd, P., Desimone, R., \& Ungerleider, L. G. (1998). Mechanisms of directed attention in the human extrastriate cortex as revealed by functional MRI. Science, 282, 108-111.

MiLLER, J. (1981). Global precedence in attention and decision. Journal of Experimental Psychology: Human Perception \& Performance, 7,1161-1174.

NAvON, D. (1977). Forest before trees: The precedence of global features in visual perception. Cognitive Psychology, 9, 353-383.

Pomerantz, J. R. (1983). Global and local precedence: Selective attention in form and motion perception. Journal of Experimental Psychology: General, 112, 516-540.

PoSNER, M. I., \& KeELE, S. W. (1968). On the genesis of abstract ideas. Journal of Experimental Psychology, 77, 353-363.

RENSINK, R. A. (2002). Change detection. Annual Review of Psychology, 53, 245-277.

ReYNoLDS, J., Chelazzi, L., \& Desimone, R. (1999). Competitive mechanisms subserve attention in macaque areas V2 and V4. Journal of Neuroscience, 19, 1736-1753.

Robertson, L. C., Egly, R., LAMB, M. R., \& Kerth, L. (1993). Spatial attention and cuing to global and local levels of hierarchical structure. Journal of Experimental Psychology: Human Perception \& Performance, 19, 471-487.

SiMONs, D. J., \& LEvin, D. T. (1997). Change blindness. Trends in Cognitive Sciences, 1, 261-267.

TeGHTSOONIAn, M. (1965). The judgment of size. American Journal of Psychology, 78, 392-402.

TrEISMAN, A. (1969). Strategies and models of selective attention. Psychological Review, 76, 282-299.

TrEISMAN, A. (1991). Search, similarity, and integration of features between and within dimensions. Journal of Experimental Psychology: Human Perception \& Performance, 17, 652-676.

TREISMAN, A., \& GoRMICAN, S. (1988). Feature analysis in early vision: Evidence from search asymmetries. Psychological Review, 95, 1548.

Treisman, A., \& Souther, J. (1985). Search asymmetry: A diagnostic for preattentive processing of separable features. Journal of Experimental Psychology: General, 114, 285-310.

WARD, L. M. (1982). Determinants of attention to local and global features of visual forms. Journal of Experimental Psychology: Human Perception \& Performance, 8, 562-581.

WATAMANiUK, S. N. J., \& McKeE, S. P. (1998). Simultaneous encoding of direction at a local and global scale. Perception \& Psychophysics, 60, 191-200.

WATAMANIUK, S. N. J., \& SeKuler, R. (1992). Temporal and spatial integration in dynamic random-dot stimuli. Vision Research, 32, 23412347.

Woods, R. L., \& Thomson, W. D. (1993). A comparison of psychometric methods for measuring the contrast sensitivity of experienced observers. Clinical Vision Science, 8, 401-415.

(Manuscript received April 21, 2003; revision accepted for publication December 19, 2003.) 